|IIIIIIIIIIIIIIIIIIIIIIIIIIIIIIIIIII

Original Article

IIIIIIIIIIIIIIIIIIIIIIIIIIIIIIIIIIIII

\title{
Enhanced production of nojirimycin via Streptomyces ficellus cultivation using marine broth and inhibitory activity of the culture for seeds of parasitic weeds
}

\author{
Kazuo Harada, ${ }^{1, *}$ Yurika Kurono, ${ }^{1}$ Saya Nagasawa, ${ }^{1}$ Tomoka Oda, ${ }^{1}$ Yudai Nasu, ${ }^{1}$ \\ Takatoshi WakabaYashi, ${ }^{2}$ Yukihiro Sugimoto, ${ }^{3}$ Hideyuki MatsuUra, ${ }^{1}$ \\ Satoru Muranaka, ${ }^{4, \#}$ Kazumasa Hirata ${ }^{1}$ and Atsushi Okazawa ${ }^{2, \$}$ \\ ${ }^{1}$ Applied Environmental Biology, Graduate School of Pharmaceutical Sciences, Osaka University, \\ 1-6 Yamadaoka, Suita, Osaka 565-0871, Japan \\ ${ }^{2}$ Department of Biotechnology, Graduate School of Engineering, Osaka University, \\ 2-1 Yamadaoka, Suita, Osaka, 565-0871, Japan \\ ${ }^{3}$ Applied Chemistry in Bioscience, Graduate School of Agricultural Science, Kobe University, \\ 1-1 Rokkodai, Nada-ku, Kobe, Hyogo, 657-8501, Japan \\ ${ }^{4}$ International Institute of Tropical Agriculture, Sabo Bakin Zuwo Road PMB 3112, Kano, Nigeria, \\ \#Present address: Japan International Research Center for Agricultural Sciences, \\ 1-1 Ohwashi, Tsukuba, Ibaraki, 305-8686, Japan \\ ${ }^{\S}$ Present address: Graduate School of Life and Environmental Sciences, Osaka Prefecture University, \\ 1-1 Gakuen-cho, Naka-ku, Sakai, Osaka, 599-8531, Japan
}

(Received May 31, 2017; Accepted September 21, 2017)

\begin{abstract}
Root parasitic weeds, such as Orobanche spp. and Striga spp., cause serious damage to crops. Recently, it was demonstrated that nojirimycin (NJ) selectively inhibits seed germination in these weeds. In this study, we modified the medium for Streptomyces ficellus to increase its production of $\mathrm{NJ}$ and evaluated the culture as an antiparasitic weed agent. We screened alternatives to Pharmamedia ${ }^{\mathrm{TM}}$, an additive in the original medium, and found that marine broth stimulated NJ production. Moreover, soluble starch-depleted medium could maintain S. ficellus NJ production. The NJ concentration reached $710 \mathrm{mg} / \mathrm{L}$ after four-day batch

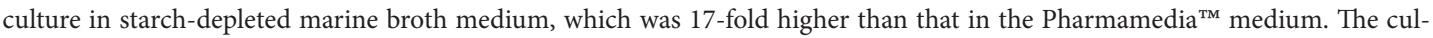
ture in the marine broth medium inhibited seed germination of Orobanche spp. and Striga spp. as effectively as a standard solution of NJ. (c) Pesticide Science Society of Japan

Keywords: marine broth, nojirimycin, parasitic weeds, Pharmamedia ${ }^{\mathrm{TM}}$, Streptomyces ficellus.

Electronic supplementary material: The online version of this article contains supplementary material (Supplemental Figure S1), which is available at http://www.jstage.jst.go.jp/browse/jpestics/
\end{abstract}

\section{Introduction}

Root parasitic weeds obtain nutrients for their growth from the roots of other plants. Orobanche spp. and Striga spp. in particular cause serious damage to agricultural crops. Orobanche spp. is distributed from the tropics to the subarctic and is commonly located around the Mediterranean and in the Middle East. It parasitizes a wide range of crops including Leguminosae, Solanaceae, and Asteraceae. Striga spp. grows in the semiarid tropics, especially in Africa and South Asia, and lives on hosts such

\footnotetext{
* To whom correspondence should be addressed.

E-mail: harada6@phs.osaka-u.ac.jp

Published online October 26, 2017

(c) Pesticide Science Society of Japan
}

as sorghum, maize, and millet, which are staple crops of these regions. It is estimated that Striga spp. affects 50 million hectares of farmland and 300 million farmers, resulting in damage valued at approximately seven billion dollars each year. ${ }^{1,2)}$

Seeds of root parasitic weeds receive germination stimulants, such as strigolactones, from host plant roots, after which their elongated radicles invade the host roots. ${ }^{3,4)}$ By the time the weeds appear above ground, they have already seriously damaged the host plants. Therefore, it is important to remove the seeds of parasitic weeds from farms and inhibit their germination, elongation, and/or invasion. However, a single individual parasitic plant can generate over 100,000 seeds, each of which is very small (approximately $0.2-0.4 \mathrm{~mm}$ ). Consequently, it is very difficult to remove the seeds from farms. Researchers have previously suggested various methods for controlling parasitic weeds. 
For example, suicide germination has been induced by synthetic germination stimulants. ${ }^{5)}$ Host cultivars that secrete decreased levels of strigolactones have also been developed. ${ }^{6}$ However, these methods do not completely inhibit the parasites; thus, an effective method has not yet been developed.

Recently, Wakabayashi et al. demonstrated that nojirimycin (NJ) selectively inhibits seed germination and radicle elongation in parasitic weeds. ${ }^{7} \mathrm{NJ}$ constitutes an antibiotic that is classified as an azasugar, which primarily inhibits $\beta$-glycosidase. ${ }^{8-11)} \mathrm{NJ}$ was originally discovered in a Streptomyces species culture isolated from soil collected in Hokkaido, Japan. ${ }^{12)}$ Streptomyces species isolated in other areas, such as Nagano, Japan and Taiwan, also produce NJ. Previous studies have reported a method of producing NJ using Streptomyces ficellus and Pharmamedia ${ }^{\mathrm{TM}}$, a supplement prepared from cotton and often used for secondary metabolite production by actinomycetes. ${ }^{13-15)}$ However, the production efficiency of $\mathrm{NJ}$ is low, whereas it needs to be produced on a large scale for use as an antiparasitic weed agent. In addition, if the culture medium that contains NJ does not require purification and can be used to directly inhibit seed germination and/or radicle elongation in parasitic weeds with efficiency, production costs will be reduced.

Our objective was to develop an NJ-containing antiparasitic weed agent. Our specific aims were to modify the S. ficellus culture medium to increase NJ production and evaluate the value of the culture.

\section{Materials and Methods}

\section{Materials}

Reagents used in this study were purchased from Nacalai Tesque (Japan) unless otherwise specified. Streptomyces ficellus (JCM catalogue No. 4946) was provided by the Japan Collection of Microorganisms, RIKEN BRC, as a participant in the National BioResource Project of the MEXT, Japan. The strain was recovered from glycerol stocks, and the culture was subcultured three times for NJ production. The medium contained $2.0 \mathrm{~g} / \mathrm{L}$ yeast extract (BD Bacto ${ }^{\mathrm{TM}}$ Yeast Extract, Becton, Dickinson and Company, Franklin Lakes, NJ, USA) and $10 \mathrm{~g} / \mathrm{L}$ soluble starch, and the $\mathrm{pH}$ was adjusted to 7.3 with sodium hydroxide. Seeds of Orobanche minor and Striga gesnerioides were collected from mature plants parasitizing red clover in Yokohama, Japan, and cowpeas in Kano, Nigeria, respectively. Seeds of Striga hermonthica were collected from mature plants in a sorghum field near Wad Medani and supplied by Professor Babiker, Sudan University of Science and Technology, Sudan.

\section{NJ production culture}

$S$. ficellus cultivation for NJ production was performed in a medium containing $13.6 \mathrm{~g} / \mathrm{L}$ glucose, $40 \mathrm{~g} / \mathrm{L}$ soluble starch, $8.0 \mathrm{~g} / \mathrm{L}$ calcium carbonate, $20 \mathrm{~g} / \mathrm{L}$ blackstrap molasses (EM Seikatsu, Nagoya, Japan), and $25 \mathrm{~g} / \mathrm{L}$ additive reagent, adjusted to $\mathrm{pH}$ 7.2. For screening an effective additive reagent for NJ production, either BD Bacto ${ }^{\mathrm{TM}}$ Tryptic Soy Broth, BD Difco ${ }^{\mathrm{TM}}$ Marine Broth 2216, BD Difco ${ }^{\mathrm{TM}}$ Mueller Hinton Broth, Pharmamedia ${ }^{\mathrm{TM}}$
(Archer Daniels Midland Company, Chicago, IL, USA), polypeptone (Wako Pure Chemical Industries, Osaka, Japan), BD Difco $^{\mathrm{TM}}$ Skim Milk, BD Bacto ${ }^{\mathrm{TM}}$ Tryptone, or BD Bacto ${ }^{\mathrm{TM}}$ Yeast Extract was used. The 20-times-diluted subculture was inoculated into fresh medium and cultured at $28^{\circ} \mathrm{C}$ and $170 \mathrm{rpm}$. In this study, we also evaluated the effect of removing soluble starch. For analyzing the time courses of cell growth, glucose, and NJ concentrations in S. ficellus culture in the medium supplemented by marine broth, we prepared an NJ production medium without soluble starch. For analyzing the NJ stability and bioactivity of $S$. ficellus culture, we also prepared NJ production medium without soluble starch.

\section{Gas chromatography/flame ionization detection (GC/FID) and gas chromatography/mass spectrometry (GC/MS)}

The culture was filtered through a membrane filter $(0.22 \mu \mathrm{m}$ pore size, New Steradisc, KURABO, Osaka, Japan) to remove cells and any solid material. The filtrate $(50 \mu \mathrm{L})$ was transferred to a new microtube, and $50 \mu \mathrm{L}$ of $10 \mathrm{mM}$ ribitol (Wako Pure Chemicals) in distilled water was added. The solution was lyophilized overnight. The derivatization of NJ in residue was carried out with $50 \mu \mathrm{L}$ of $20 \mathrm{mg} / \mathrm{mL}$ methoxyamine hydroxychloride (Sigma-Aldrich, St. Louis, MO, USA) in pyridine at $30^{\circ} \mathrm{C}$ for $90 \mathrm{~min}$ and, subsequently, with $50 \mu \mathrm{L}$ of $N$-methyl- $N$ (trimethylsilyl)trifluoroacetamide (MSTFA) (GL Sciences, Tokyo, Japan) at $37^{\circ} \mathrm{C}$ for $30 \mathrm{~min}$. An aliquot of the derivatized sample $(1 \mu \mathrm{L})$ was subjected to gas chromatography/flame ionization detection (GC/FID) and gas chromatography/mass spectrometry (GC/MS). GC/FID was carried out using a GC4000 (GL Sciences) equipped with a $30 \mathrm{~m} \times 0.25 \mathrm{~mm}$ i.d. fused silica capillary column coated with a $0.25 \mu \mathrm{m}$ Inert Cap 5 (GL Sciences) and coupled to an ASI 240 autosampler (GL Sciences). The split ratio was $1 / 20$. The injector temperature was set at $230^{\circ} \mathrm{C}$, and the helium gas flow rate through the column was $1 \mathrm{~mL} / \mathrm{min}$. The column temperature was held at $150^{\circ} \mathrm{C}$ for $2 \mathrm{~min}$, then raised by $10^{\circ} \mathrm{C} / \mathrm{min}$ to $325^{\circ} \mathrm{C}$ and held there for $5 \mathrm{~min}$. The detection temperature was set at $320^{\circ} \mathrm{C}$. GC/MS was performed using a JEOL AutoMass (Tokyo, Japan) equipped with a $30 \mathrm{~m} \times 0.25 \mathrm{~mm}$ i.d. fused silica capillary column coated with $0.25 \mu \mathrm{m}$ Agilent J\&W HP-5MS (Agilent Technologies, Santa Clara, CA, USA), and connected to a deactivated fused silica capillary $(0.25 \mathrm{~mm}$ i.d. $\times 1 \mathrm{~m}$, GL Sciences) as a guard column. The split ratio was $1 / 50$, and the helium gas flow rate through the column was $0.8 \mathrm{~mL} / \mathrm{min}$. The injector and column temperatures were the same as for the GC analysis. Electron ionization was carried out using a $70 \mathrm{eV}$ electron beam at $280^{\circ} \mathrm{C}$. Mass spectra were recorded at a $2 / \mathrm{s}$ scan rate in the mass range of $\mathrm{m} / z$ 40-700.

\section{Monitoring cell growth and glucose consumption}

For monitoring cell growth during NJ production, $250 \mu \mathrm{L}$ of culture was diluted with $750 \mu \mathrm{L}$ of the subculture medium. The mixture was centrifuged at $750 \times g$ for $5 \mathrm{~min}$. The cell pellet was washed with $1 \mathrm{~mL}$ of the subculture medium and centri- 
fuged again. Then, $1 \mathrm{~mL}$ of the subculture medium was added to the pellet and vortexed. An aliquot of $250 \mu \mathrm{L}$ of the cell suspension was transferred to a new microtube and diluted with $750 \mu \mathrm{L}$ of the subculture medium. A total of $200 \mu \mathrm{L}$ of the diluted suspension was transferred to a new microtube, and $40 \mu \mathrm{L}$ of 3-(4,5-dimethylthiazol-2-yl)-5-(3-carboxymethoxyphenyl)-2(4-sulfophenyl)-2H-tetrazolium (MTS) reagent (Promega, Madison, WI, USA) was added, following incubation at $25^{\circ} \mathrm{C}$ for $1 \mathrm{hr}$ in the dark. Then, the UV absorbance at $490 \mathrm{~nm}$ was recorded. Glucose concentrations in the culture were measured with an F-kit for D-glucose (Roche Diagnostics GmbH, Mannheim, Germany), using culture that had been diluted 10 times with distilled water.

\section{Bioactivity assay for parasitic weed germination}

The seed germination assay was conducted as described previously. ${ }^{7,16)}$ Seeds of parasitic weeds were surface sterilized with a solution containing $1 \%(\mathrm{v} / \mathrm{v})$ sodium hypochlorite and $0.1 \%$ (v/v) Tween ${ }^{\circledR} 20$ for $2 \mathrm{~min}$, rinsed with distilled water, and dried under vacuum. For O. minor, approximately 50 surface-sterilized seeds were conditioned on a filter disk $(10 \mathrm{~mm}$, Whatman GF/D; GE Healthcare, Uppsala, Sweden) that was placed on another filter paper $(47 \mathrm{~mm}$, Whatman GF/D) in a Petri dish $(50 \mathrm{~mm})$ with $1.5 \mathrm{~mL}$ distilled water and kept in the dark at $23^{\circ} \mathrm{C}$ for one week. A total of four filter disks with seeds were placed in each Petri dish. S. hermonthica and S. gesnerioides seeds were similarly conditioned at $30^{\circ} \mathrm{C}$ for $10-12$ days on $8 \mathrm{~mm}$ glass fiber filter paper disks. After conditioning, GF/D disks with seeds were transferred to a new Petri dish containing fresh filter paper ( $47 \mathrm{~mm}$, Whatman GF/D) to remove the surplus water. Germination of $O$. minor was induced by adding $1.5 \mathrm{~mL}$ of $0.5 \mathrm{mg} / \mathrm{L}$ (w/v) rac-GR24 solution to the filter paper. S. hermonthica seeds were induced by adding $20 \mu \mathrm{L}$ of $0.5 \mathrm{mg} / \mathrm{L}$ (w/v) rac-GR24 solution to each $8 \mathrm{~mm}$ glass fiber disk, whereas $S$. gesnerioides seeds induced by cowpea root exudates were prepared as described previously. ${ }^{16)}$ The effect of test solutions on seed germination was evaluated. The test solutions were i) water, ii) the NJ standard solution, iii) NJ production medium supplemented by marine broth and starch-depleted prior to inoculation with S. ficellus (MMB), and iv) the inoculated culture with marine broth (CMB) collected 4 days after inoculation with S. ficellus. The solution of iii) was used to evaluate the effects of the other components except for $\mathrm{NJ}$ on germination. Each solution was filtrated through a membrane filter $(0.22 \mu \mathrm{m}$ pore size, New Steradisc, KUBOTA), mixed with the rac-GR24 solution or cowpea root exudates, and then applied to seeds. The treated O. minor seeds were incubated at $23^{\circ} \mathrm{C}$ and microscopically evaluated after 6 days for germination (radicle protrusion). The seeds of $S$. hermonthica and S. gesnerioides were incubated at $30^{\circ} \mathrm{C}$ and then examined for germination after 24 and $48 \mathrm{hr}$, respectively.

\section{Results}

To improve NJ production by S. ficellus, we modified the growth medium described by Argoudelis and Reusser. ${ }^{15)}$ Pharmame-
$\mathrm{dia}^{\mathrm{TM}}$, an additive used in their medium, was substituted with conventional medium components. The cultures were sampled four days after inoculation and analyzed by GC/FID. At a retention time of $11.9 \mathrm{~min}$, an NJ peak that was similar to the NJ standard peak was detected in the inoculated culture with marine broth (MB in Fig. 1). In the GC/MS analysis of the NJ standard solution, NJ had a retention time of $9.6 \mathrm{~min}$ and fragmentation ions at $m / z 188,204,244,285,304$, and 375. A peak with the same fragmentation ions at the same retention time was detected in the culture in the marine broth medium (Fig. 2); this peak was not detected in the marine broth medium prior to inoculation with S. ficellus (Fig. 2).

Next, we analyzed time course profiles of cell growth, glucose, and NJ concentrations in the cultures. The medium for NJ production contained blackstrap molasses and insoluble materials; accordingly, optical density measurements and cell collection were difficult. Therefore, cell growth was measured using an MTS assay. In order to evaluate the effects of not only substitution of Pharmamedia ${ }^{\mathrm{TM}}$ with marine broth, but also starch depletion, we compared the time courses of the S. ficellus cultures in four types of NJ production media: i) supplemented by Pharmamedia $^{\mathrm{TM}}$ (Fig. S1 A), ii) supplemented by marine broth (Fig. S1 B), iii) supplemented by Pharmamedia ${ }^{\mathrm{TM}}$ and depleted soluble starch (Fig. S1 C), and iv) supplemented by marine broth and depleted soluble starch (Fig. S1 D). Supplemental Figure S1 shows the time course profiles of these cultures. We did not observe significant effects of starch depletion. Figure 3 summarizes the time courses of the S. ficellus cultures in two types of NJ production media; one was supplemented by Pharmamedia ${ }^{\mathrm{TM}}$,

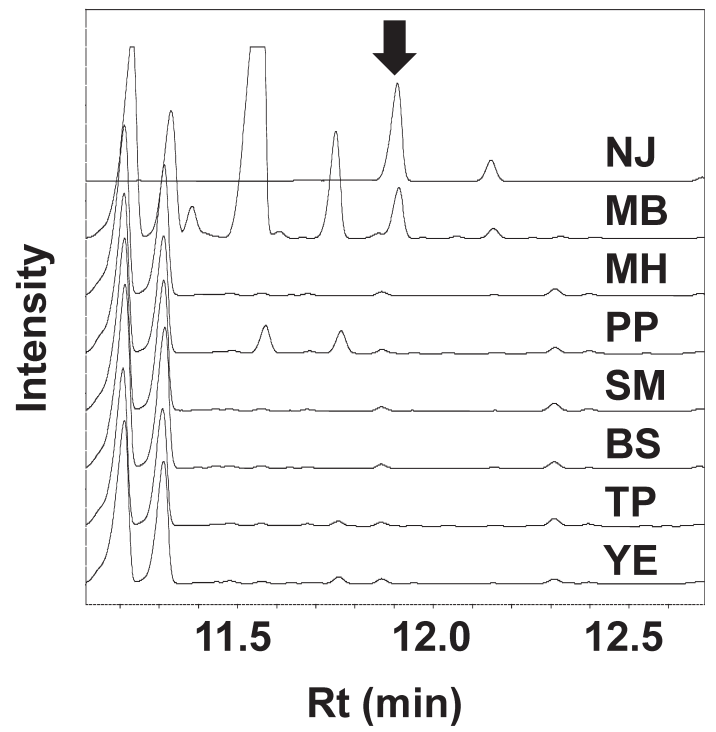

Fig. 1. Gas chromatography/flame ionization detection overlaid on chromatograms of Streptomyces ficellus cultures. The arrow indicates nojirimycin. Samples were collected four days after inoculation. NJ indicates the nojirimycin standard (10 mM). MB, MH, PP, SM, BS, TP, and YE indicate $S$. ficellus cultures in NJ production media supplemented by marine broth, Muller Hinton Broth, Polypeptone, Skim Milk, Bacto Tryptic Soy Broth, Tryptone, and Yeast Extract, respectively. 

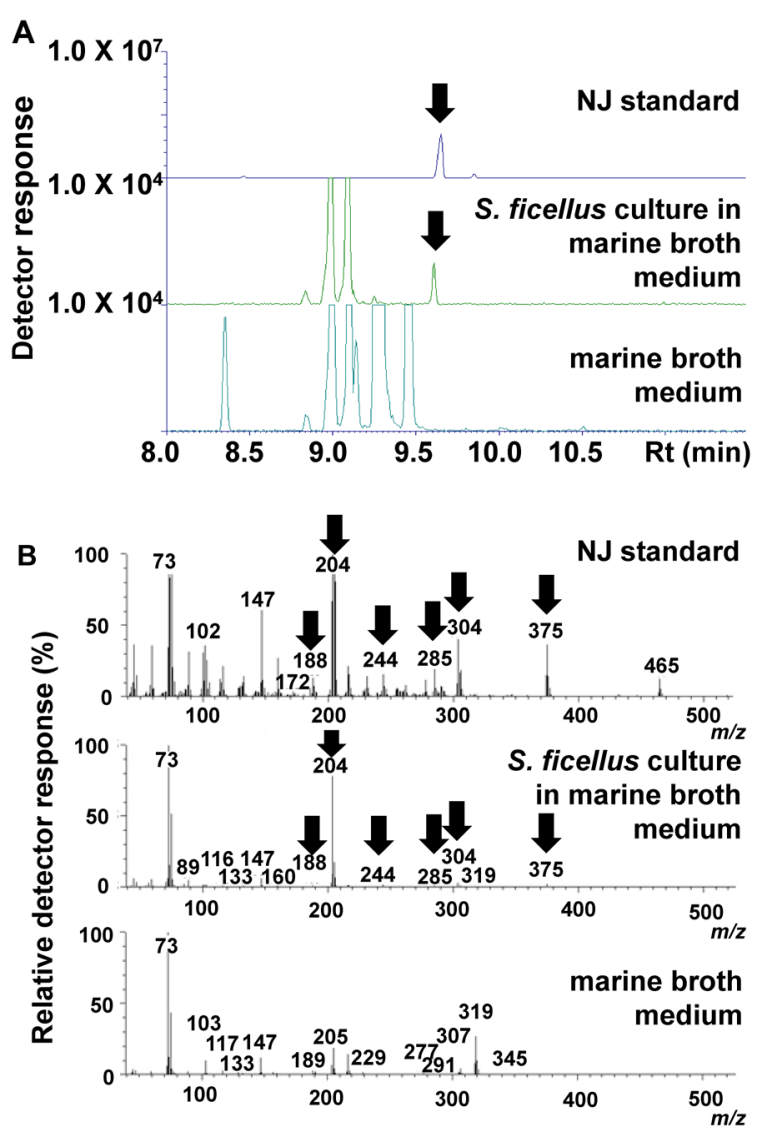

Fig. 2. Results of gas chromatography/mass spectrometry analyses of nojirimycin standard solution, Streptomyces ficellus culture in NJ production medium supplemented by marine broth, and NJ production medium supplemented by marine broth prior to inoculation with $S$. ficellus. (A) Mass chromatograms of $\mathrm{m} / z$ 375. Arrows indicate NJ. (B) Mass spectra at $9.6 \mathrm{~min}$. Arrows show peaks detected in the NJ standard and S. ficellus culture in NJ production medium supplemented by marine broth, although not prior to inoculation with S. ficellus.

and the other was supplemented by marine broth without soluble starch. In the Pharmamedia ${ }^{\mathrm{TM}}$-supplemented culture, MTS activity was observed after 1 day and remained steady until 14 days (Fig. 3A). The glucose concentration decreased and was depleted at 6 days after inoculation (Fig. 3B). The NJ concentration increased slightly after inoculation, reached $42 \mathrm{mg} / \mathrm{L}$ at 4 days, and then decreased (Fig. 3C), whereas in the marine broth-supplemented and starch-depleted culture, MTS activity was observed at 2 days and then slowly decreased (Fig. 3A). The glucose concentration decreased very slowly and was depleted at 13 days (Fig. 3B). The NJ concentration peaked at 4 days with a concentration of $710 \mathrm{mg} / \mathrm{L}$, which was 17 -fold higher than in the Pharmamedia ${ }^{\mathrm{TM}}$-supplemented culture (Fig. 3C). It should be noted that the $S$. ficellus culture in the NJ production medium supplemented by marine broth and depleted starch collected from 4-day culture was, therefore, used for subsequent experiments, abbreviated as CMB in the current manuscript.

Next, we evaluated the stability of $\mathrm{NJ}$ in $\mathrm{CMB}$ at different storage temperatures (Fig. 4). At $4^{\circ} \mathrm{C}, 100 \%$ of the $\mathrm{NJ}$ remained after
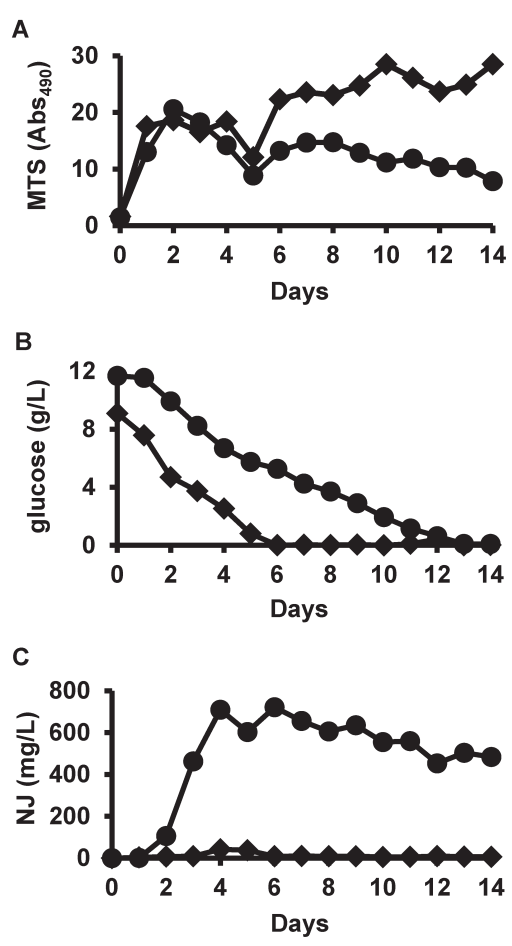

Fig. 3. Time course profiles of cell amounts and glucose and nojirimycin (NJ) concentrations in Streptomyces ficellus cultures. Of the two types of NJ production media tested, one was supplemented by Pharmamedia ${ }^{\text {тм }}$ (symbol: diamond), and the other was supplemented by marine broth without soluble starch (symbol: circle). The time courses of cell amounts (A), glucose concentration (B), and NJ concentration (C) are shown. Cell amounts were determined by a 3-(4,5-dimethylthiazol-2-yl)-5-(3carboxymethoxyphenyl)-2-(4-sulfophenyl)-2H-tetrazolium (MTS) assay. F-kit and gas chromatography/flame ionization detection were used to measure glucose and NJ concentrations, respectively.

three months and $73 \%$ remained after six months. At $25^{\circ} \mathrm{C}, 53 \%$ and $40 \%$ of the NJ remained after 14 days and one month, respectively. At $40^{\circ} \mathrm{C}, 51 \%$ and $2 \%$ of the NJ remained after three days and one month, respectively. Over $50^{\circ} \mathrm{C}$, NJ decreased to below $10 \%$ in three days (data not shown). At -80 and $-30^{\circ} \mathrm{C}$,

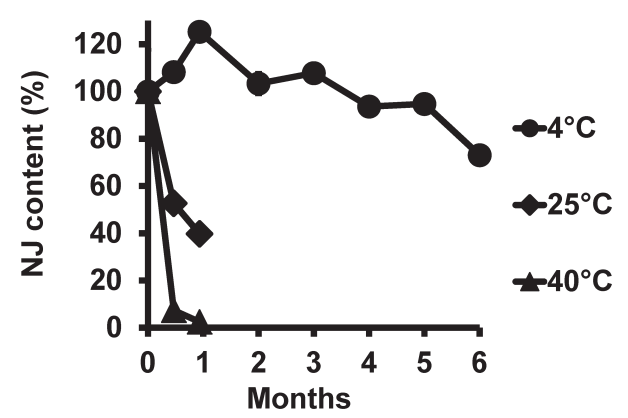

Fig. 4. Time course of nojirimycin (NJ) concentrations in S. ficellus culture in NJ production medium supplemented by marine broth and depleted starch collected from 4 -day culture $(\mathrm{CMB})$ during storage at $4^{\circ} \mathrm{C}, 25^{\circ} \mathrm{C}$, and $40^{\circ} \mathrm{C}$. NJ concentrations were determined by gas chromatography/ flame ionization detection. The Y-axis shows the concentration relative to the initial one. 
A Orobanche minor

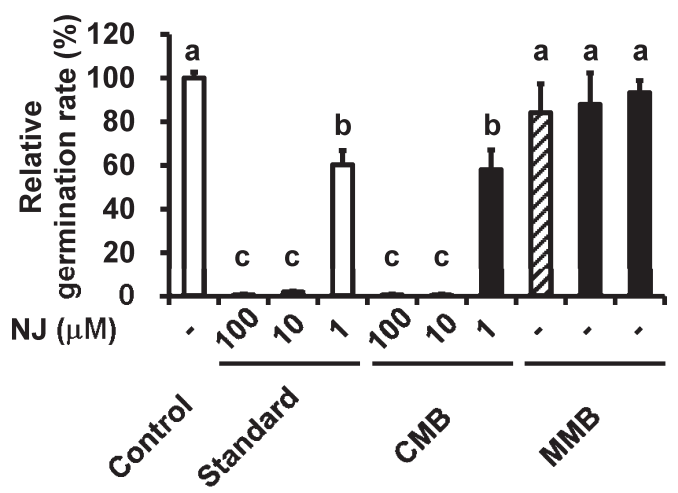

B Striga gesnerioides

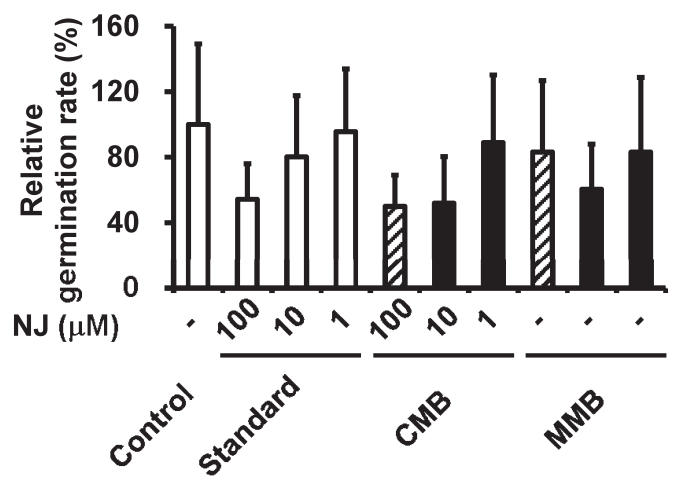

C Striga hermonthica

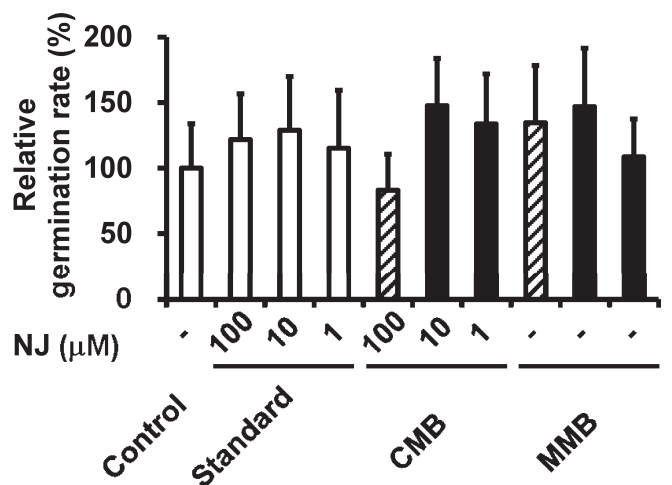

Fig. 5. Germination rates of parasitic weed seeds treated with nojirimycin (NJ) standard solution, S. ficellus culture in NJ production medium supplemented by marine broth and depleted starch collected from 4-day culture (CMB), and NJ production medium supplemented by marine broth and depleted starch prior to inoculation with S. ficellus (MMB). All seeds were treated with germination stimulants (rac-GR24, Orobanche minor and Striga hermonthica; cowpea exudate, Striga gesnerioides). Control seeds were only treated with germination stimulants, whereas the others were treated with germination stimulants and $\mathrm{NJ}$ standard solution, CMB or MMB. CMB was diluted to adjust the NJ concentration, as indicated along the $\mathrm{X}$-axis. $\mathrm{MMB}$ and $\mathrm{CMB}$ bars with the same fill pattern indicate the same dilutions. Values are relative to the samples treated with only germination stimulants (control) and are presented as the means \pm S.D. $(n=3-5)$. The values along the $\mathrm{X}$-axis indicate the NJ concentrations $(\mu \mathrm{M})$ in the treatment solutions. Different lowercase letters indicate significant differences between treatments (Tukey-Kramer, $p<0.05$ ).
NJ contents showed a similar pattern as at $4^{\circ} \mathrm{C}$ (data not shown).

The inhibitory effect of $\mathrm{CMB}$ on parasitic weed germination was subsequently evaluated. We adjusted NJ concentrations in $\mathrm{CMB}$ to 1,10 , or $100 \mu \mathrm{M}$ by dilution with distilled water. We also measured the activity of $\mathrm{NJ}$ standard solutions $(1,10$, and $100 \mu \mathrm{M})$ and diluted NJ production medium supplemented by marine broth without starch prior to inoculation of S. ficellus (MMB, same dilution rates) as references. MMB did not inhibit the germination of $O$. minor seeds, whereas $\mathrm{CMB}$ containing 10 or $100 \mu \mathrm{M}$ of NJ completely inhibited germination. Moreover, the relative germination rate for $\mathrm{CMB}$ containing $1 \mu \mathrm{M}$ of $\mathrm{NJ}$ to a control treated with only a germination stimulant was $58 \%$. The inhibition observed with CMB was identical to that seen with the same concentration of an NJ standard solution (Fig. $5 \mathrm{~A})$.

For S. gesnerioides seeds, CMB containing 10 or $100 \mu \mathrm{M}$ of NJ inhibited germination by $52 \%$ or $50 \%$, respectively, as compared with a control. Relative germination rates to the control decreased by $80 \%$ and $54 \%$ with 10 and $100 \mu \mathrm{M}$, respectively, of the NJ standard solutions. Germination decreased slightly after treatment with $\mathrm{MMB}$ that was diluted by the same volume as $\mathrm{CMB}$ containing $10 \mu \mathrm{M} \mathrm{NJ}$; however, it was not affected by MMB diluted by other volumes (Fig. 5B). Germination of $S$. hermonthica was not inhibited by any level of the NJ standards or CMB (Fig. 5C).

\section{Discussion}

This study demonstrated that marine broth significantly increased NJ production by S. ficellus. It also showed that removing soluble starch from the growth medium did not affect NJ production. Table 1 presents the NJ production and costs of the original medium and our optimized medium. At 4 days, CMB contained $710 \mathrm{mg} / \mathrm{L}$ of NJ, a level that was 17 -fold higher than in Pharmamedia ${ }^{\mathrm{TM}}$-supplemented culture $(42 \mathrm{mg} / \mathrm{L})$. Based on current (Dec. 2016) market prices, the unit costs per $1 \mathrm{~kg}$ are 17.60 USD (glucose), 6.30 USD (black strap molasses), 42.70 USD (calcium carbonate), 67.10 USD (soluble starch), 81.0 USD (Pharmamedia ${ }^{\mathrm{TM}}$ ), and 387.00 USD (marine broth). At these prices, the material costs for $1 \mathrm{~L}$ of Pharmamedia ${ }^{\mathrm{TM}}$ - and marine broth-supplemented medium are 3.60 USD and 10.40 USD, respectively. The volumes of Pharmamedia ${ }^{\mathrm{TM}}$ - and marine broth-supplemented medium required for $1 \mathrm{~g}$ of $\mathrm{NJ}$ production

Table 1. Nojirimycin production costs.

\begin{tabular}{lcc}
\hline & Pharmamedia $^{\mathrm{TM}}$ & marine broth \\
\hline $\begin{array}{l}\text { NJ production (mg/L after four-day } \\
\text { culture) }\end{array}$ & 42 & 710 \\
NJ production ratio (Pharmamedia ${ }^{\mathrm{TM}}$ ) & 1.0 & 17 \\
Cost of 1 L of medium (USD) & 3.6 & 10.4 \\
$\begin{array}{l}\text { Volume of medium for 1 g of NJ } \\
\text { production (L) }\end{array}$ & 23.8 & 1.4 \\
$\begin{array}{l}\text { Cost of medium for 1g of NJ produc- } \\
\text { tion (USD) }\end{array}$ & 86.7 & 14.6 \\
\hline
\end{tabular}


are $23.6 \mathrm{~L}$ and $1.4 \mathrm{~L}$, respectively. Therefore, the Pharmamedia ${ }^{\mathrm{TM}}$ and marine broth-supplemented medium costs for $1 \mathrm{~g}$ of $\mathrm{NJ}$ production are 86.70 USD and 14.60 USD, respectively. Consequently, using marine broth-supplemented medium reduces the overall production cost by $83 \%$.

Commercial NJ is sold as a sulfite adduct, which makes it more stable. Wakabayashi et al. demonstrated that the NJ sulfite adduct inhibits parasitic weed seed germination. ${ }^{7)}$ If S. ficellus cultures could be stored for a long duration without any treatment, such as sulfite adduction, and still exhibit inhibitory activity, costs might be further reduced. This study, therefore, also evaluated NJ stability and inhibitory activity against the seed germination of parasitic weeds. NJ in CMB was stable for three months below $4^{\circ} \mathrm{C}$. Therefore, we could store $\mathrm{CMB}$ with high activity for three months. Conversely, $\mathrm{NJ}$ in $\mathrm{CMB}$ at $25^{\circ} \mathrm{C}$ quickly lost activity. This indicates that $\mathrm{NJ}$ would not linger as a residue in host plants and soil. As the seed germination of parasitic weeds is completed within several days after stimulation, this suggests that several days of NJ application should be effective for weed control.

$\mathrm{NJ}$ in $\mathrm{CMB}$ inhibited the germination of O. minor seeds and also inhibited the germination of $S$. gesnerioides seeds. Notably, the inhibition seen with $\mathrm{CMB}$ containing NJ was the same as that observed using NJ standards. Therefore, $\mathrm{CMB}$ might be used as an antiparasitic weed agent without purification. Although CMB did not inhibit S. hermonthica germination, it tended to inhibit radicle elongation (data not shown). The inhibition of radicle elongation was also observed upon treatment with an NJ standard. ${ }^{7)}$ Parasitic weeds cannot survive unless they parasitize host plants immediately after germination; thus, the inhibition of radicle elongation represents a promising method of controlling parasitic weeds. The components in CMB except for NJ would not contribute to these inhibitory activities.

The composition of marine broth is similar to that of seawater, containing high levels of sodium and chloride ions; accordingly, it is used for cultivating heterotrophic marine bacteria. Based on the manufacturer's catalog, sodium and chloride concentrations were estimated as $7.8 \mathrm{~g} / \mathrm{L}$ and $17.7 \mathrm{~g} / \mathrm{L}$, respectively. Therefore, if CMB is applied to crops without dilution, it would cause salt damage. However, a 100-fold dilution of CMB enabled us to completely inhibit $O$. minor germination; thus, diluted $\mathrm{CMB}$ can be used as a pesticide against O. minor. In comparison, the addition of the same amount of sodium chloride to the culture instead of marine broth did not increase NJ production by S. ficellus (data not shown). This indicated that sodium and chloride ions themselves did not increase NJ production, nor did the change in osmotic pressure. Additional studies are required to identify the NJ synthesis inducer(s) in marine broth to determine whether a low-salt alternative might be formulated.
In conclusion, we developed an effective medium for NJ production by S. ficellus, the key component of which was marine broth. We also demonstrated that the culture did not require purification to inhibit the germination of parasitic weed seeds. The results of this study will likely contribute to the development of effective herbicides for parasitic weeds.

\section{Acknowledgements}

We greatly appreciate the help of Munenori Suzuki and Toshiya Muranaka (Department of Bioengineering, Graduate School of Engineering, Osaka University, Japan) for the GC/MS analyses. We also thank Abdel Gabbar T. Babiker (Sudan University of Science and Technology, Sudan) for the supply of S. hermonthica seeds, and Hiroshi Kinoshita (International Center for Biotechnology, Osaka University, Japan) for technical advice. This study was supported by a research grant from the Institute for Fermentation, Osaka (to K. H.) and a grant for Industrial Technology Research (Financial Support for Young Researchers, 09E52502a) from the New Energy and Industrial Technology Development Organization (NEDO), Japan to K. H., S. M., and A. O.

\section{References}

1) A. Elzein and J. Kroschel: FAO Corporate Document Repository, http://www.fao.org/docrep/006/y5031e/y5031e0a.htm) (Accessed on 19th Oct., 2017)

2) C. Parker: Pest Manag. Sci. 65, 453-459 (2009).

3) J. A. López-Ráez, R. Matusova, C. Cardoso, M. Jamil, T. Charnikhova, W. Kohlen, C. Ruyter-Spira, F. Verstappen and H. Bouwmeester: Pest Manag. Sci. 65, 471-477 (2009).

4) K. Yoneyama, X. Xie, K. Yoneyama and Y. Takeuchi: Pest Manag. Sci. 65, 467-470 (2009).

5) B. Zwanenburg, A. S. Mwakaboko, A. Reizelman, G. Anilkumar and D. Sethumadhavan: Pest Manag. Sci. 65, 478-491 (2009).

6) D. E. Hess, G. Ejeta and L. G. Butler: Phytochemistry 31, 493-497 (1992).

7) T. Wakabayashi, B. Joseph, S. Yasumoto, T. Akashi, T. Aoki, K. Harada, S. Muranaka, T. Bamba, E. Fukusaki, Y. Takeuchi, K. Yoneyama, T. Muranaka, Y. Sugimoto and A. Okazawa: J. Exp. Bot. 66, 30853097 (2015).

8) S. Inouye, T. Tsuruoka and T. Niida: J. Antibiot. (Tokyo) 19, 288-292 (1966).

9) N. Ishida, K. Kumagai, T. Niida, K. Hamamoto and T. Shomura: J. Antibiot. (Tokyo) 20, 62-65 (1967).

10) N. Ishida, K. Kumagai, T. Niida, T. Tsuruoka and H. Yumoto: J. Antibiot. (Tokyo) 20, 66-71 (1967).

11) T. Niwa, S. Inouye, T. Tsuruoka, Y. Koaze and T. Niida: Agric. Biol. Chem. 34, 966-968 (1970).

12) T. Nishikawa and N. Ishida: J. Antibiot. (Tokyo) 18, 132-133 (1965).

13) A. D. Argoudelis, F. Reusser, H. A. Whaley, L. Baczynskyj, S. A. Mizsak and R. J. Wnuk: J. Antibiot. (Tokyo) 29, 1001-1006 (1976).

14) A. D. Argoudelis, F. Reusser, S. A. Mizsak and L. Baczynskyj: J. Antibiot. (Tokyo) 29, 1007-1014 (1976).

15) A. D. Argoudelis and F. Reusser: US Patent 3998698 A, (1976).

16) K. Ueno, M. Fujiwara, S. Nomura, M. Mizutani, M. Sasaki, H. Takikawa and Y. Sugimoto: J. Agric. Food Chem. 59, 9226-9231 (2011). 